\title{
Polyaniline/Polystyrene Blends: In-Depth Analysis of the Effect of Sulfonic Acid Dopant Concentration on AC Conductivity Using Broadband Dielectric Spectroscopy
}

\author{
Noora Al-Thani, Mohammad K. Hassan (D), and Jolly Bhadra \\ Center for Advanced Materials, Qatar University, P.O. Box 2713, Doha, Qatar \\ Correspondence should be addressed to Mohammad K. Hassan; mohamed.hassan@qu.edu.qa
}

Received 2 January 2018; Accepted 25 February 2018; Published 4 April 2018

Academic Editor: Renbo Wei

Copyright (c) 2018 Noora Al-Thani et al. This is an open access article distributed under the Creative Commons Attribution License, which permits unrestricted use, distribution, and reproduction in any medium, provided the original work is properly cited.

\begin{abstract}
This work presents an in-depth analysis of the alternating current (AC) conductivity of polyaniline-polystyrene (PANI-PS) blends doped with camphor sulfonic acid (CSA) and prepared using an in situ dispersion polymerization technique. We prepared the blends using fixed ratios of PS to PANI while varying the concentration of the CSA dopant. The AC conductivity of the blends was investigated using broadband dielectric spectroscopy. Increasing CSA resulted in a decrease in the AC conductivity of the blends. This behaviour was explained in terms of the availability of a lone pair of electrons of the NH groups in the polyaniline, which are typically attacked by the electron-withdrawing sulfonic acid groups of CSA. The conductivity is discussed in terms of changes in the dielectric permittivity storage $\left(\varepsilon^{\prime}\right)$, loss $\left(\varepsilon^{\prime \prime}\right)$, and modulus $\left(M^{\prime \prime}\right)$ of the blends over a wide range of temperatures. This is linked to the glass transition temperature of the PANI. Dielectric spectra at low frequencies indicated the presence of pronounced MaxwellWagner-Sillars (MWS) interfacial polarization, especially in samples with a low concentration of CSA. Electrical conduction activation energies for the blends were also calculated using the temperature dependence of the direct current (DC) conductivity at a low frequency $\left(\sigma_{\mathrm{dc}}\right)$, which exhibit an Arrhenius behaviour with respect to temperature. Scanning electron microscopy revealed a fibrous morphology for the pure PANI, while the blends showed agglomeration with increasing CSA concentrations.
\end{abstract}

\section{Introduction}

In the last few decades, intensive research has focused on intrinsic electrically conductive polymers (ICP) because of their possible applications in light-emitting diodes, batteries, electromagnetic shielding, antistatic coating, and gas sensors [1-5]. Lack of processability in the doped state hinders the ability of ICPs to be blended with industrial polymers. Because of a synergic combination of mechanical properties of conventional insulating polymers with electrical properties of the conducting polymers, blending is of great technological importance. In terms of the percolation threshold of the conductive materials in any blend, polymeric materials have better properties than the inorganic conductive phase like carbon black or metal particles.

In the conducting polymers family, polyaniline (PANI) has excellent tunable electrical conductivity desired for electrical and electronic industries. Upon protonation by Lewis acid, these polymers can be switched to a wide conductivity range [6]. Since the last two decades, a lot of researches have been carried out to improve the PANI processability by maintaining the electrical properties. Different methods of composite preparation, such as blending, doping with acids, and copolymerization, have been adopted to obtain processable PANI, and these efforts have resulted in many research publications [7-10]. PANI in a modified, processable form has wide applications in the electrical and electronics industries [11, 12].

Various methods currently exist to make PANI processable. These include doping with organic acids, such as dodecyl benzene sulfonic acid (DBSA) [13] or camphor sulfonic acid (CSA) [14]. These sulfonated acids have long tails that enable the PANI to dissolve in nonpolar or weakly polar common organic solvents, which can cosolubilize most commercial polymers. This method has been utilized to prepare a number of PANI blends in which both PANI 
complexes and preferred polymers are soluble in the same solvent. PANI has poor mechanical properties and it is generally procured in powder form. Of various processes used, blending of PANI with other polymers (thermoplastic or thermosetting) is one of the most commonly used techniques to enhance its mechanical strength. Using this method, the synergic combination of improved electrical properties from PANI and enhanced mechanical properties from conventional polymers can be amalgamated to produce a material with more potential applications in the electronics industry [15]. Blending of PANI with different types of conventional polymers, such as polyacrylonitrile [16], polyvinyl alcohol (PVA), and polystyrene (PS) $[17,18]$, have been investigated. PS has some useful features, such as good thermal stability, a degradation temperature above $400^{\circ} \mathrm{C}$, good chemical resistance, and significant mechanical toughness.

Dielectric spectroscopy, also known as impedance spectroscopy (IS), is a powerful tool to study the effects of interactions between electromagnetic radiation and the chemical or physical properties of the materials $[19,20]$. All matter consists of molecules and atoms with electric charges that respond whenever an electric field is applied to them. These responses are in the form of translational or rotational motion and they give rise to macroscopic electrical or dielectric behaviours in the material. Information regarding dielectric behaviours is useful for a variety of fields, such as electrical engineering, physical chemistry, electronic physics, and material science. Thus, using impedance spectroscopy, we can efficiently study polymer chain motion and different dispersion modes of fillers inside a polymer blend, which are related to the chemical composition, molecular structure, and phase morphology.

In order to investigate the electrical transport properties of any complex system, impedance spectroscopy (IS) is one of the most convenient tools used in solid state electronic system [21, 22]. In our previous works, we studied effect of sulfonic acid dopants on the CSA-doped PANI-PS blends for the gas sensor application [6]. This study concluded that the gas sensitivity and reversibility of the blends have a strong correlation with their conductivity. However, not many details on the electrical transport properties were done in our previous work. Therefore, herein and as a continuation to our prior study, we use broadband dielectric spectroscopy to present a detailed, in-depth investigation of the electrical transport behaviour of CSA-doped into PANI-PS blends and how this correlates with CSA concentrations.

\section{Experimental}

2.1. Experimental Section. The polymers and chemicals used for experiments were acquired from Merck and Sigma chemical companies and these were of very high purity (99.9\%). Further purification of aniline was performed by repeated distillation in a vacuum following preservation at a low temperature prior to use. The oxidant (ammonium persulphate), toluene, and acid (CSA) were used as obtained. During the synthesis, all solutions were prepared using double-distilled water.
PANI was obtained using dispersion polymerization with conventional techniques described previously [23, 24]. A solution of aniline and CSA in aqueous medium was prepared by stirring them for 1 hour. Following a polymerization step, ammonium persulphate was added dropwise with constant stirring. The whole system was maintained at a temperature of $0-5^{\circ} \mathrm{C}$. Upon completion of the oxidant addition process, the reaction was constantly stirred for an additional 24 hours. The reaction turned into a bluish-green homogeneous mixture, indicating the completion of the polymerization reaction. The resulting precipitate was then filtered and washed with deionized water and methanol until the filtrate became colourless to remove any oligomer or unreacted oxidant. PANI powder was then dried under reduced pressure at $40^{\circ} \mathrm{C}$ for 24 hours. The PANI-PS blend was prepared by following the above process except for replacing water and using predissolved PS in toluene by stirring for 1 hour instead. Four different concentrations of dopants $(0.2 \mathrm{M}, 0.3 \mathrm{M}, 0.4 \mathrm{M}$, and $0.5 \mathrm{M}$ ) were used for the blend preparation. Finally, the blend solution was used to cast films on glass slides and interdigitate finger electrode thick film (gold, $15 \mathrm{~mm} \times$ $15 \mathrm{~mm}$ ) on an alumina substrate ( $250 \mu \mathrm{m}$ online/spaces $)$.

2.2. Characterization Techniques. Dielectric measurements were performed using a Novocontrol GmbH Concept 40 broadband dielectric spectrometer, and the data were collected over the frequency range $0.1 \mathrm{~Hz}-3 \mathrm{MHz}$ at fixed temperatures in the range of -70 to $180^{\circ} \mathrm{C}$. Samples were prepared and dried directly on $40 \mathrm{~mm}$ diameter stainless steel electrodes, and another $20 \mathrm{~mm}$ diameter stainless steel electrode was used as a top electrode during testing. The AC conductivity was calculated with the Novocontrol Win DETA software by using the measured values of dielectric permittivity and the dielectric loss factor.

The morphologies of the synthesized CSA-doped into PANI-PS blends were observed with scanning electron microscopy (SEM) using a nano-SEM Nova 450. In order to detect the glass transition temperatures of the composites, differential scanning calorimetry (DSC) tests were performed using a Perkin Elmer DSC 8500.

\section{Results and Discussion}

3.1. Dielectric Spectroscopy Measurements. Broadband dielectric spectroscopy is a powerful tool to interrogate polymeric material characteristics because both the conductivity and chain motion can be monitored in the same spectra. However, in the case of samples containing conducting polymers, such as PANI, the dielectric loss $\left(\varepsilon^{\prime \prime}\right)$ and storage $\left(\varepsilon^{\prime}\right)$ spectra are dominated by electrode polarization and conductivity effects [25-28]. In this case, conductivity overwhelms the relaxation peaks related to the glass transition and other secondary motions, which makes their analysis difficult. The conductivity engulfing pattern is clearly indicated by the high values of $\varepsilon^{\prime}$ and $\varepsilon^{\prime \prime}$ at different temperatures (Figures 1(a)1(b)). Consequently, researchers suggested using complex electric modulus $M^{*}(\omega)$ and conductivity $\sigma^{*}(\omega)$ functions to 


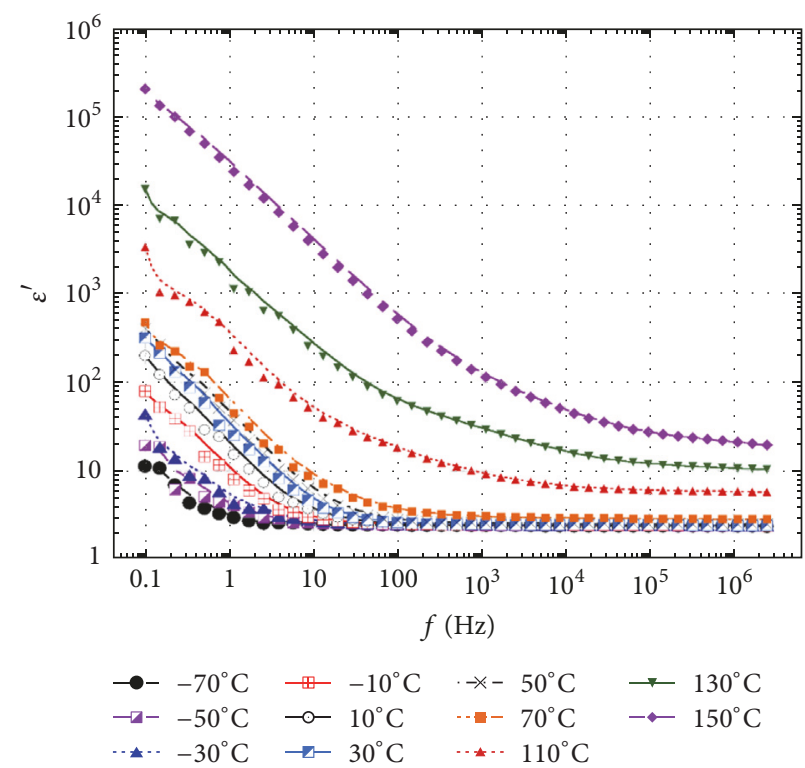

(a)

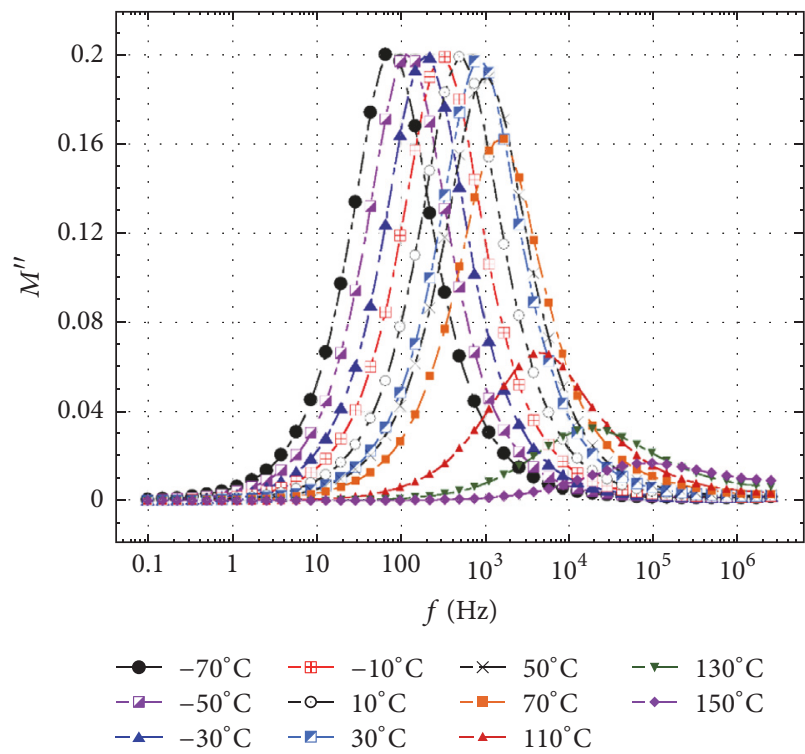

(c)

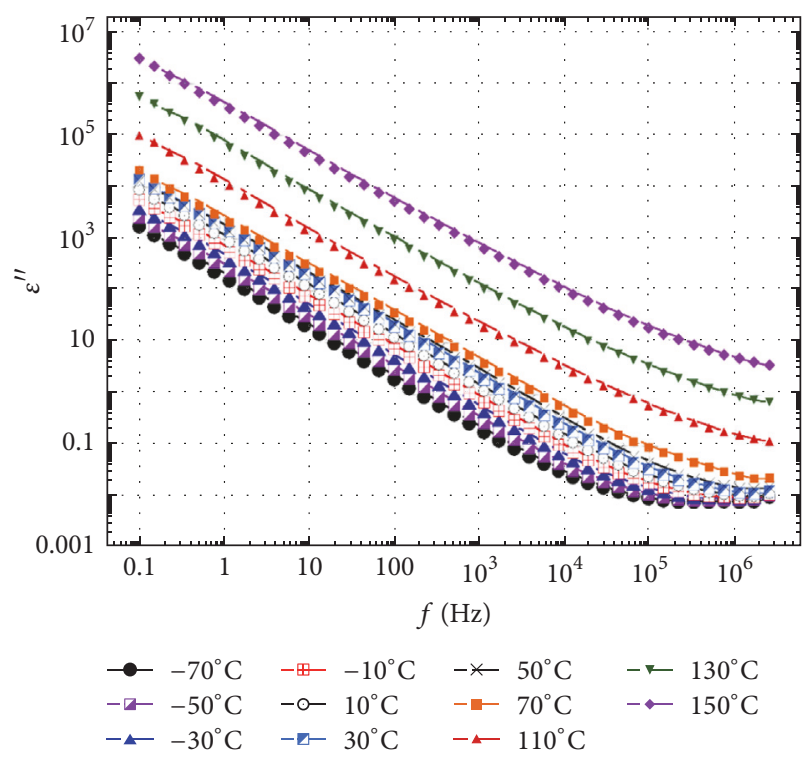

(b)

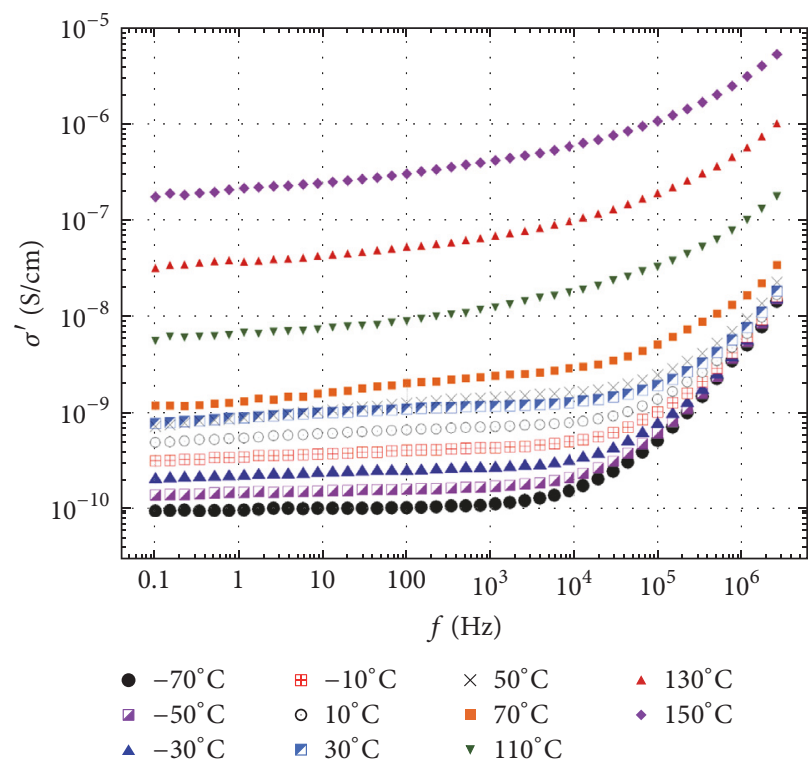

(d)

Figure 1: Dielectric permittivity storage (a), loss (b), electrical modulus (c), and AC conductivity (d) for the PANI-PS-0.2 CSA sample at different temperatures.

analyse the dielectric spectra of the conductive materials [2529]. These functions are related via the equation:

$$
M^{*}(\omega)=\frac{1}{\varepsilon^{*}(\omega)}=\frac{1}{\left(\varepsilon^{\prime}-i \varepsilon^{\prime \prime}\right)}=\frac{i \varepsilon_{o} \omega}{\sigma^{*}(\omega)},
$$

where $\sigma^{*}=\sigma^{\prime}+i \sigma^{\prime \prime}\left(\sigma^{\prime}=\varepsilon_{o} \omega \varepsilon^{\prime \prime} ; \sigma^{\prime \prime}=\varepsilon_{o} \omega \varepsilon^{\prime}\right)$ and $M^{*}=$ $M^{\prime}+i M^{\prime \prime}\left(M^{\prime}=\varepsilon^{\prime} /\left(\varepsilon^{\prime 2}+\varepsilon^{\prime \prime 2}\right) ; M^{\prime \prime}=\varepsilon^{\prime \prime} /\left(\varepsilon^{\prime 2}+\varepsilon^{\prime \prime 2}\right)\right)$.

The conductivity relaxation peak shifts to lower frequencies as the temperature decreases, indicating slower charge motion (Figure 1(c)). Additionally, the intensity of the $M^{\prime \prime}$ relaxation peak increases with cooling and reaches a constant value once the glass transition of PANI (around $\left.40^{\circ} \mathrm{C}\right)[30,31]$ is reached. No other relaxation modes are evident in the $M^{\prime \prime}$ spectra of this sample (PANI-PS-0.2 CSA). The AC conductivity $\left(\sigma^{\prime}\right)$ of the PANI-PS-0.2 CSA sample is shown (Figure $1(\mathrm{~d})$ ). AC conductivity exhibits a typical behaviour for polymers and disordered solids. It has frequency independence at low frequencies and then upsurges monotonically with the $s$-the power of frequency, where $s$ ranges from 0.7 to 1.0 [32]. Conductivity plateaus at a low frequency corresponding to the DC conductivity, and the 
frequency at which it becomes frequency-dependent is called the relaxation frequency, $\omega_{s}$. DC conductivity increases and the plateau widens to include the entire frequency range at higher temperatures.

In order to study the contribution of the CSA dopant to the electron transport performance of the samples, comparative $3 \mathrm{D}$ isochronal diagrams $\left(M^{\prime \prime}\right.$ versus temperature and frequency, $f$ ) for the all-PANI-PS blends and pure PANI films were developed (Figure 2). Two relaxations were noted: namely, the conductivity and the PANI or PS $T_{g}$-related peaks. The pure PANI and 0.2 M CSA-doped samples exhibited strong conductivity relaxation and some features around $50^{\circ} \mathrm{C}$, which could be related to the longrange $T_{g}$ motions of the PANI and PS chains. In these two samples, high conduction resulting from multiple electron hopping events completely overwhelmed the polarizability attained from the dipolar chain motion. However, in the 0.3-0.5 M CSA-doped samples, electron hopping events were comparatively low, and the conduction relaxation peaks were weaker compared to those arising from the dipolar chain motions. This change in behaviour associated with increased concentrations of CSA dopant in the blends was further illustrated by the dielectric permittivity loss $\left(\varepsilon^{\prime \prime}\right)$ versus $f$ spectra at $20^{\circ} \mathrm{C}$. Very high $\varepsilon^{\prime \prime}$ values at low $f$ were observed for pure PANI, and a rapid drop as the CSA content increased in the blend was evident (Figure 3). High values of $\varepsilon^{\prime \prime}$ at a low $f$ corresponded to the DC electrical conductivity, $\sigma_{\mathrm{dc}}$, which resulted from free charge polarization within the material [33]. This charge polarization completely overwhelmed the PANI polymer chain relaxation, which was evident for the samples containing $0.3 \mathrm{M}, 0.4 \mathrm{M}$, and $0.5 \mathrm{M}$ CSA.

AC conductivity behaviour at $20^{\circ} \mathrm{C}$ for all samples was examined (Figure 4). The DC conductivity increased, and the plateau widened to include most of the measured frequency ranges for only pure PANI and the 0.2 M CSA-doped samples. For CSA concentrations above $0.2 \mathrm{M}$, the conductivity did not show any plateaus and its gradual increase with increasing frequency was mainly due to the motion of the PANI and PS chain dipoles (relaxation region) [34]. The temperature dependence of DC conductivity indicated that the observed metallic DC conductivity was due to only a small fraction of delocalized carriers, which are primarily present in metallic islands. Furthermore, this is the achievable conductivity for such systems when the entire charge carrier density participates are very high [6]. It has also been shown that the relaxation of the charge carrier system is attributed to the charge hopping of mobile carriers, which can lead to both short-range $\sigma_{\mathrm{ac}}$ and long-range $\sigma_{\mathrm{dc}}$. Saravanan et al. reported the attachment of sulfonic acid group to the lone pair of electrons on the $\mathrm{NH}$ groups in the polyaniline chain without altering the aromatic ring (Figure 5) [19]. Therefore, increasing PANI loading should increase AC conductivity, which was reported by Belaabed et al. for the PANI/epoxy composites. This is because of two reasons. First, increasing the PANI content will lead to an increased charge carrier concentration, making it easier for better interparticle contact and, thus, the formation of a conducting network. Second, differences in the dielectric constant and conductivity of PANI and the surrounding epoxy matrix
TABLE 1: Conduction activation energies for the pure PANI and PANI-PS blends containing various concentrations of CSA.

\begin{tabular}{lcc}
\hline Samples & Activation energy $(\mathrm{eV})$ & $R^{2}$ value \\
\hline Pure PANI & 0.023 & 0.9847 \\
PANI-PS-CSA-0.2 & 0.055 & 0.9877 \\
PANI-PS-CSA-0.3 & 0.587 & 0.9816 \\
PANI-PS-CSA-0.4 & 0.521 & 0.9829 \\
PANI-PS-CSA-0.5 & 0.721 & 0.9629 \\
\hline
\end{tabular}

may lead to the Maxwell-Wagner-Sillars (MWS) interfacial polarization effect because of the accumulation of mobile charges at the interfaces between the two matrices [34, 35]. PANI dipole density, mobility of the dipoles, and the mobility of the surrounding matrix polymer chains are some of the important parameters required for the MWS polarization phenomena to occur. In our system, however, the amount of PANI is fixed for all samples, and the CSA content is the only variable among the composite samples. Therefore, the availability of the $\mathrm{NH}$ groups' electrons is the only parameter that governs the conductivity behaviour exhibited in Figure 4.

The temperature dependence of the dc DC conductivity $\left(\sigma_{\mathrm{dc}}\right)$ is presented (Figure 6). Plateau values of the AC conductivity at low frequency (Figure 4) were used to obtain the $\sigma_{\mathrm{dc}}$ values, which accounted for the long-range hopping of the charge carriers of the NH groups [36, 37]. Charge carriers typically accomplish more simple hops before the applied electric field reverses at low frequency, resulting in the DC conduction effect. These hops are also facilitated above the matrix $T_{g}$ because of the increased segmental mobility of the polymer chains, which causes the charge carriers to become more mobile [33]. The observed temperature dependence of $\sigma_{\mathrm{dc}}$ (Figure 6) exhibited Arrhenius behaviour according to the equation:

$$
\sigma_{\mathrm{dc}}=\sigma_{o} \exp \left(-\frac{E_{a}}{k_{B} T}\right),
$$

where $\sigma_{o}$ is the per-exponent factor, $k_{B}$ is the Boltzmann constant, and $E_{a}$ is the activation energy for the conduction process. Conduction activation energy values were calculated from the slopes of the linear correlations (Figure 6, Table 1). The conduction activation energy is the minimum energy required to overcome the potential barrier in polymeric systems. As expected, pure PANI had the lowest activation energy, and the values increased with increasing concentrations of CSA in the blends, confirming the attachment of sulfonic acid groups to the electrons of the $\mathrm{NH}$ groups, which was noted above. This low energy barrier for pure PANI and the 0.2 M CSA-doped samples reflected metallic or narrow band-gap semiconducting behaviour and it was close to the values previously reported for other conductive polymer composites [37]. Only these two samples showed DC conduction, which was indicated by the plateau in the $\sigma^{\prime}$ versus the $f$ plots over a wide range of temperatures starting at $-70^{\circ} \mathrm{C}$. However, samples with higher CSA doping started to show this type of conduction only at $50^{\circ} \mathrm{C}$, which is greater than the $T_{g}$ of polyaniline [30,31]. 

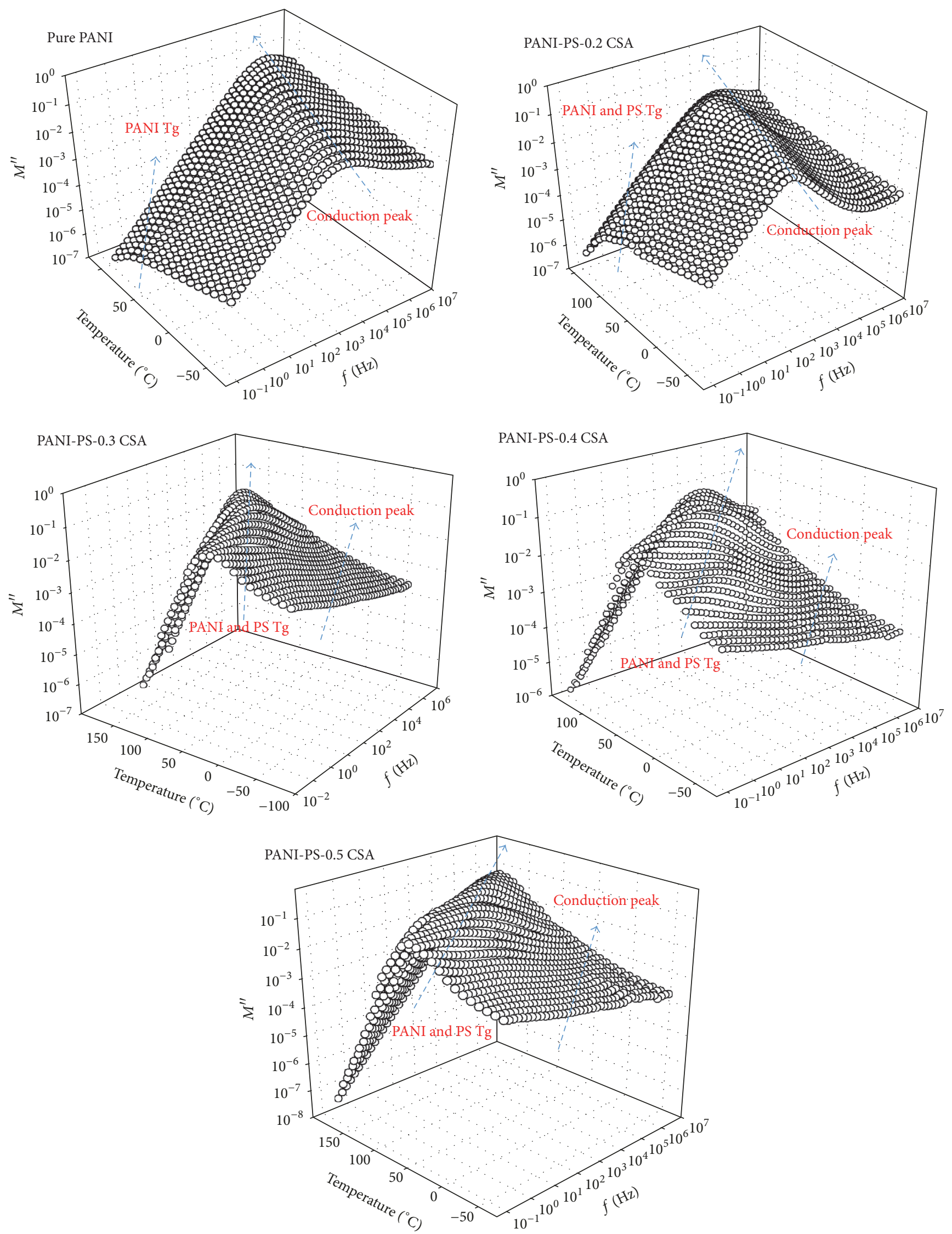

FIGURE 2: 3D plots of the electrical modulus versus temperature and frequency for the samples. 


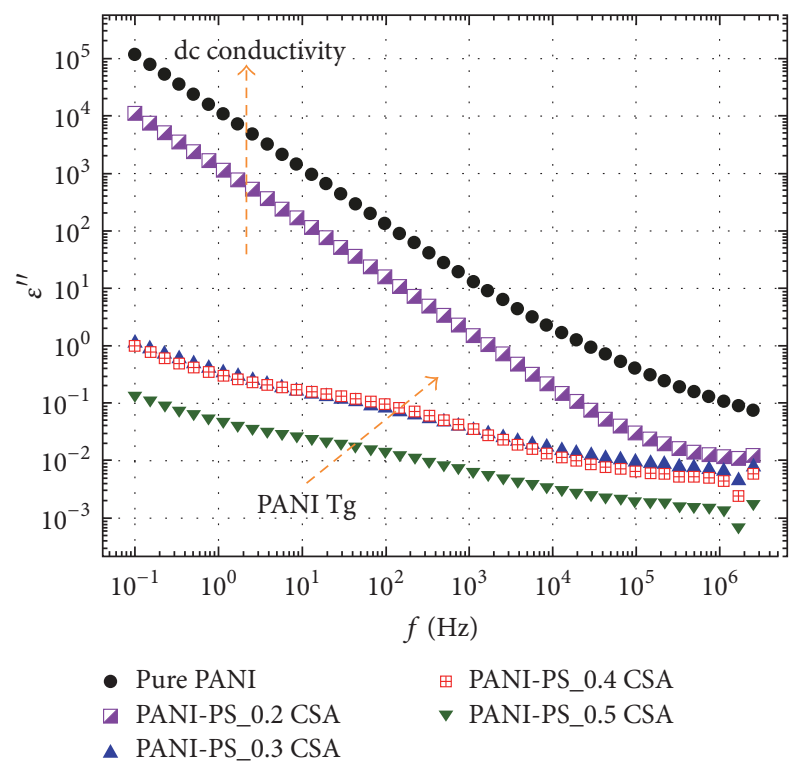

FIGURE 3: $\varepsilon^{\prime \prime}$ versus $f$ at $20^{\circ} \mathrm{C}$ for the pure PANI and PANI-PS blends containing variable CSA concentrations.

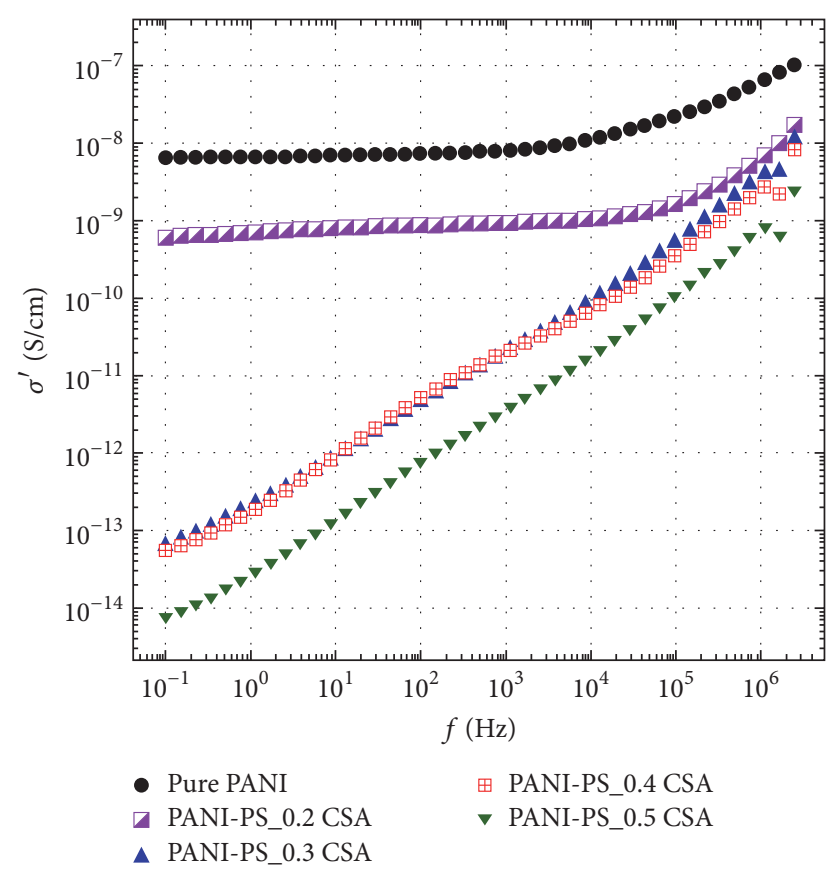

FIgURe 4: AC conductivity versus frequency at $20^{\circ} \mathrm{C}$ for pure PANI and PANI-PS blends samples with different composition.

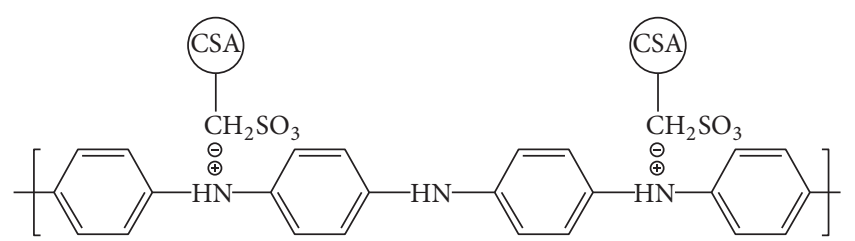

FIGURE 5: Interaction of the camphor sulfonic acid with polyaniline.

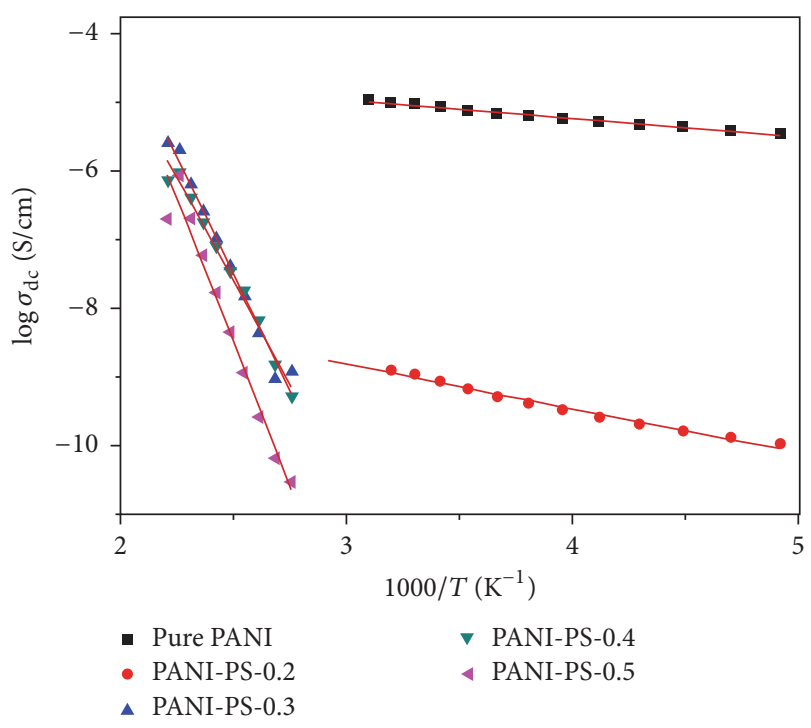

FIgURE 6: Temperature dependence of $\sigma_{\mathrm{dc}}$ for the pure PANI and PANI-PS blends of different compositions.

3.2. SEM Analysis. SEM was performed on the pure PANI and four CSA-doped PANI-PS blends (Figure 7). The pure PANI sample displayed a fibrous morphology of nanodimension, whereas the blends showed some agglomeration that increased with higher dopant concentrations. A chemical blending method was used for sample preparation of PANI and PS for the miscible and phased materials.

3.3. DSC Analysis. A DSC analysis was used to examine the changes in glass transition temperatures of the blends as the CSA concentrations varied. Because not many significant changes were seen for all the steps except the second heating of the DSC thermogram, only the second heating curves are shown (Figure 8). No $T_{g}$ was observed for pure $\mathrm{HCl}$-doped PANI as expected from the literature $[30,31]$. Nevertheless, in all the CSA-doped PANI-PS blends, we observed $T_{g}$ at about $90^{\circ} \mathrm{C}$, which was also observed in the dielectric results above. There was not much difference in $T_{g}$ with regard to the concentration of the dopant in the blends.

\section{Conclusion}

Broadband dielectric spectroscopy was used to examine the AC conductivity of PANI-PS blends containing different concentrations of the CSA dopant. The electrical conduction response of the samples strongly correlated with the CSA concentration in the blend. Samples containing only pure PANI or a small concentration of CSA showed very high values for dielectric loss $\left(\varepsilon^{\prime \prime}\right)$ and storage $\left(\varepsilon^{\prime}\right)$, which suggested the existence of electrode polarization and Maxwell-Wagner-Sillars interfacial polarization effects, especially at low frequencies and higher temperatures. These samples clearly display strong conductivity relaxation, which overwhelmed the dipolar relaxation associated with the long-range glass transitionrelated motions of the PANI and PS chains. However, as the CSA concentration increased, the conduction relaxation 


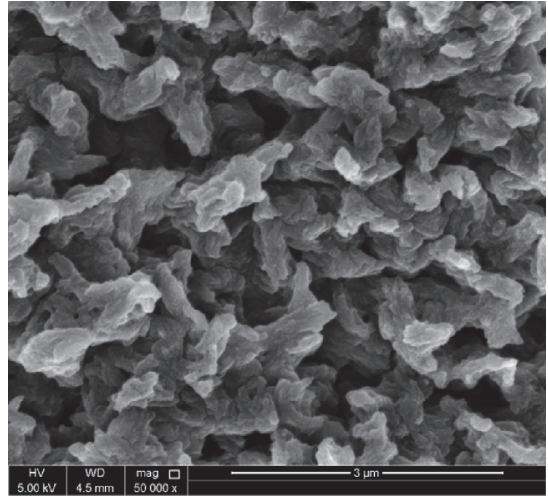

(a)

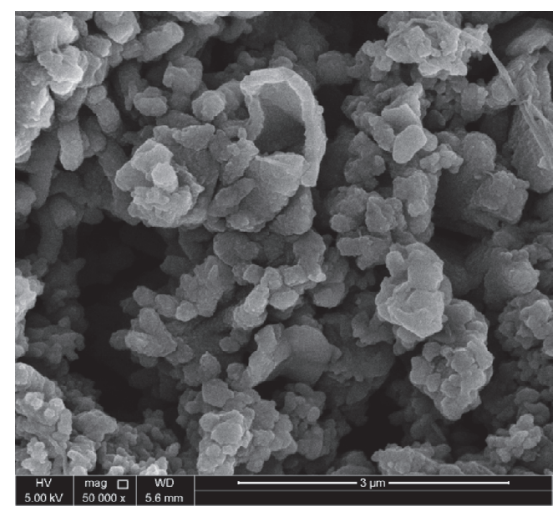

(b)

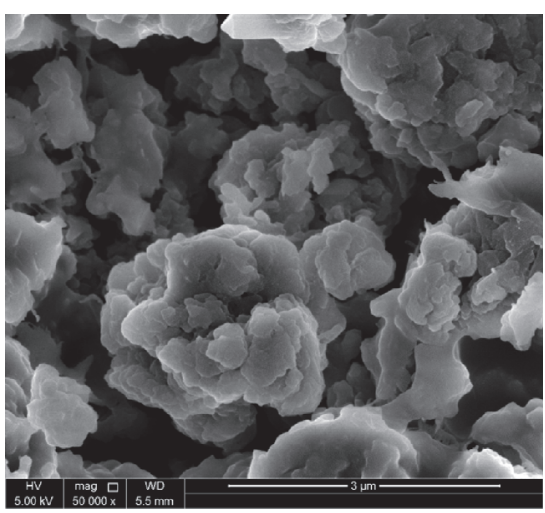

(c)

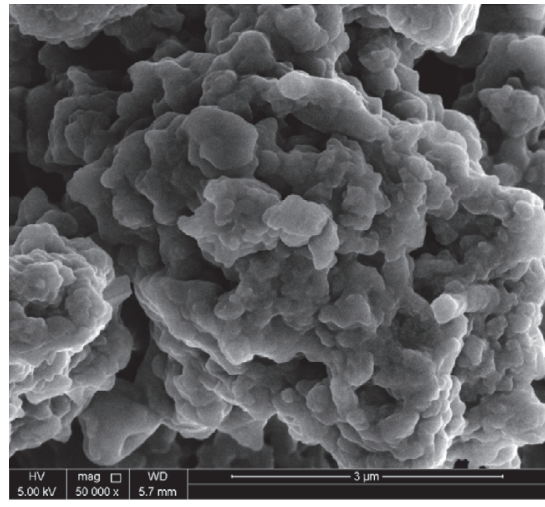

(d)

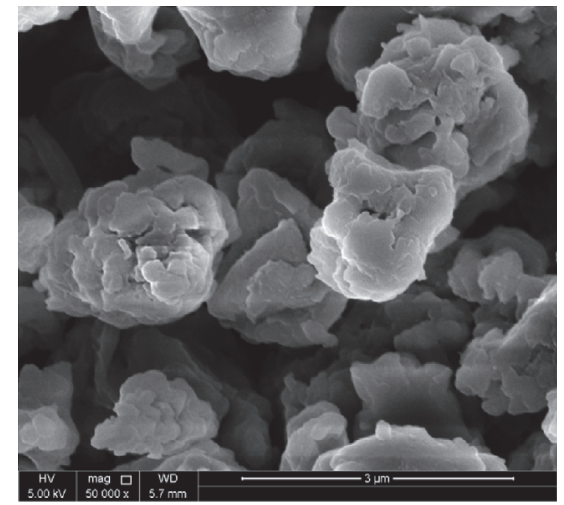

(e)

FIgure 7: SEM images of pure PANI and CSA-doped PANI-PS blends. (a) Pure PANI, (b) PANI-PS 0.2 M CSA, (c) PANI-PS 0.3 M CSA, (d) PANI-PS 0.4 M CSA, and (e) PANI-PS 0.5 M CSA.

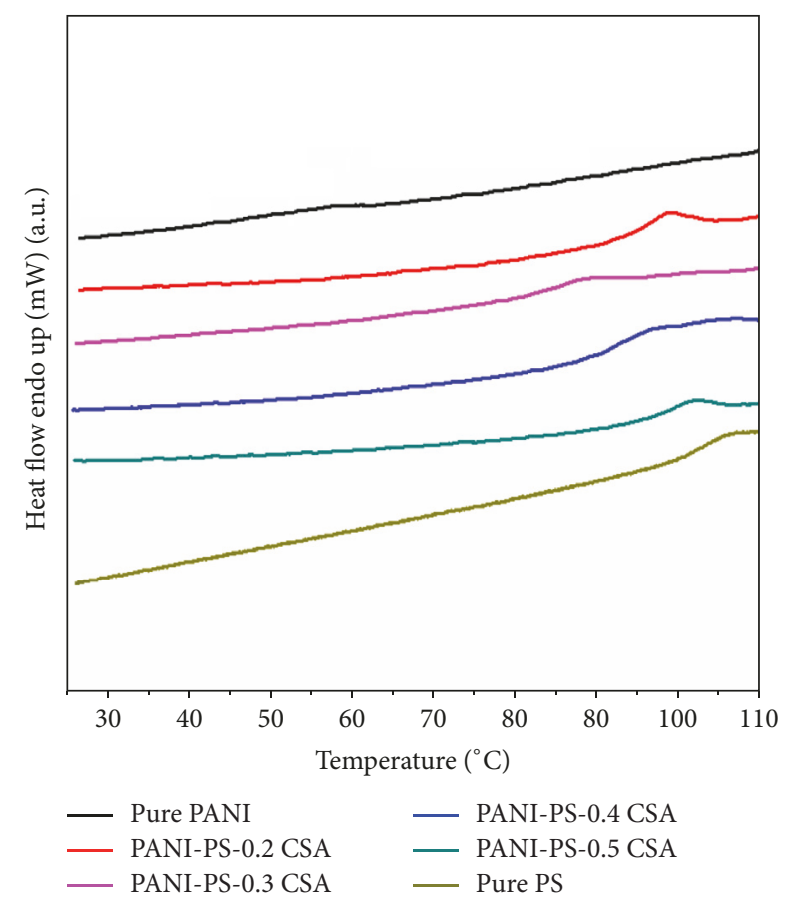

FIGURE 8: DSC analysis of the pure PANI and PANI-PS blends. peaks became weaker and could be easily distinguished from the dipolar chain motion peaks. This is thought to be due to the unavailability of the electron pairs of the $\mathrm{NH}$ groups, which become progressively attached to the increased population of the electron-withdrawing sulfonic acid groups at higher CSA concentrations. All samples displayed a strong Arrhenius behaviour for the $\log \sigma_{\mathrm{dc}}$ versus temperature plots with different values of activation energies, and this correlates well with the concentration of the CSA in the blends.

\section{Data Availability}

The data used to support the findings of this study are available from the corresponding author upon request.

\section{Conflicts of Interest}

The authors declare that they have no conflicts of interest.

\section{Acknowledgments}

The authors would like to acknowledge the financial support of the Qatar University Grant no. QUST-CAM-SPR-2017-6. 


\section{References}

[1] K. C. Persaud, "Polymers for chemical sensing," Materials Today, vol. 8, no. 4, pp. 38-44, 2005.

[2] Y. S. Negi and P. V. Adhyapak, "Development in polyaniline conducting polymers," Journal of Macromolecular Science, vol. 42, no. 1, pp. 35-53, 2002.

[3] A. Kumar, V. Kumar, and K. Awasthi, "Polyaniline-carbon nanotube composites: preparation methods, properties, and applications," Polymer-Plastics Technology and Engineering, vol. 57, no. 2, pp. 70-97, 2017.

[4] H. Khatoon and S. Ahmad, "A review on conducting polymer reinforced polyurethane composites," Journal of Industrial and Engineering Chemistry, vol. 53, pp. 1-22, 2017.

[5] M. A. Kamarudin, S. R. Sahamir, R. S. Datta, B. D. Long, M. F. M. Sabri, and S. M. Said, "A review on the fabrication of polymerbased thermoelectric materials and fabrication methods," The Scientific World Journal, vol. 2013, Article ID 713640, 17 pages, 2013.

[6] J. Bhadra, N. J. Al-Thani, N. K. Madi, and M. A. Al-Maadeed, "Preparation and characterization of chemically synthesized polyaniline-polystyrene blends as a carbon dioxide gas sensor," Synthetic Metals, vol. 181, pp. 27-36, 2013.

[7] G. Kaur, R. Adhikari, P. Cass, M. Bown, and P. Gunatillake, "Electrically conductive polymers and composites for biomedical applications," RSC Advances, vol. 5, no. 47, pp. 37553-37567, 2015.

[8] D. Nguyen and H. Yoon, "Recent advances in nanostructured conducting polymers: from synthesis to practical applications," Polymer, vol. 8, no. 4, article 118, 38 pages, 2016.

[9] T. Kobayashi, H. Yoneyama, and H. Tamura, "Polyaniline filmcoated electrodes as electrochromic display devices," Journal of Electroanalytical Chemistry, vol. 161, no. 2, pp. 419-423, 1984

[10] M. G. Han, S. K. Cho, S. G. Oh, and S. S. Im, "Preparation and characterization of polyaniline nanoparticles synthesized from DBSA micellar solution," Synthetic Metals, vol. 126, no. 1, pp. 5360, 2002.

[11] S. Bhadra, D. Khastgir, N. K. Singha, and J. H. Lee, "Progress in preparation, processing and applications of polyaniline," Progress in Polymer Science, vol. 34, no. 8, pp. 783-810, 2009.

[12] J. Wu, Q. Li, L. Fan et al., "High-performance polypyrrole nanoparticles counter electrode for dye-sensitized solar cells," Journal of Power Sources, vol. 181, no. 1, pp. 172-176, 2008.

[13] T. S. Chew, R. Daik, and M. A. A. Hamid, "Thermal conductivity and specific heat capacity of dodecylbenzenesulfonic aciddoped polyaniline particles-water based nanofluid," Polymer, vol. 7, no. 7, pp. 1221-1231, 2015.

[14] Y. Zhou, Y. Wang, D. He et al., "Synthesis and properties of nano-polyaniline films doped with camphor sulfonic acid," Journal of Nanoscience and Nanotechnology, vol. 14, no. 5, pp. 3417-3421, 2014.

[15] W. E. Jones Jr., J. Chiguma, E. Johnson, A. Pachamuthu, and D. Santos, "Electrically and thermally conducting nanocomposites for electronic applications," Materials , vol. 3, no. 2, pp. 14781496, 2010.

[16] B. Fryczkowska, Z. Piprek, M. Sieradzka, R. Fryczkowski, and J. Janicki, "Preparation and properties of composite PAN/PANI membranes," International Journal of Polymer Science, vol. 2017, Article ID 3257043, 14 pages, 2017.

[17] J. Laska, K. Zak, and A. Proń, "Conducting blends of polyaniline with conventional polymers," Synthetic Metals, vol. 84, no. 1-3, pp. 117-118, 1997.
[18] Y. Roichman, G. I. Titelman, M. S. Silverstein, A. Siegmann, and M. Narkis, "Polyaniline synthesis: Influence of powder morphology on conductivity of solution cast blends with polystyrene," Synthetic Metals, vol. 98, no. 3, pp. 201-209, 1999.

[19] S. Saravanan, M. R. Anantharaman, and S. Venkatachalam, "Structural and electrical studies on tetrameric cobalt phthalocyanine and polyaniline composites," Materials Science and Engineering: B Advanced Functional Solid-State Materials, vol. 135, no. 2, pp. 113-119, 2006.

[20] M. Kaiser, "Electrical conductivity and complex electric modulus of titanium doped nickelzinc ferrites," Physica B: Condensed Matter, vol. 407, no. 4, pp. 606-613, 2012.

[21] G. Turky, J. R. Sangoro, M. A. Rehim, and F. Kremer, "Secondary relaxations and electrical conductivity in hyperbranched polyester amides," Journal of Polymer Science Part B: Polymer Physics, vol. 48, no. 14, pp. 1651-1657, 2010.

[22] G. Turky, S. S. Shaaban, and A. Schöenhals, "Broadband dielectric spectroscopy on the molecular dynamics in different generations of hyperbranched polyester," Journal of Applied Polymer Science, vol. 113, no. 4, pp. 2477-2484, 2009.

[23] D. K. Moon, K. Osakada, T. Maruyama, and T. Yamamoto, "Preparation of polyaniline by oxidation of aniline using $\mathrm{H}_{2} \mathrm{O}_{2}$ in the presence of an iron(II) catalyst," Macromolecular Chemistry and Physics, vol. 193, pp. 1723-1728, 1992.

[24] I. Yu. Sapurina and M. A. Shishov, "Oxidative polymerization of aniline: molecular synthesis of polyaniline and the formation of supramolecular structures," in New Polymers for Special Applications, A. De Souza Gomes, Ed., InTech, 2012.

[25] S. Saravanan, C. Joseph Mathai, M. R. Anantharaman, S. Venkatachalam, and P. V. Prabhakaran, "Investigations on the electrical and structural properties of polyaniline doped with camphor sulphonic acid," Journal of Physics and Chemistry of Solids, vol. 67, no. 7, pp. 1496-1501, 2006.

[26] N. Rajeswari, S. Selvasekarapandian, S. Karthikeyan et al., "Conductivity and dielectric properties of polyvinyl alcoholpolyvinylpyrrolidone poly blend film using non-aqueous medium," Journal of Non-Crystalline Solids, vol. 357, no. 22-23, pp. 3751-3756, 2011.

[27] N. Bohli, A. Belhadj Mohamed, V. Vignéras-Lefèbvre, and J.L. Miane, "Dielectric and rheological properties of polyaniline organic dispersions," The European Physical Journal Applied Physics, vol. 46, no. 2, Article ID 20405, 2009.

[28] R. Bosisio, C. Gorini, G. Fleury, and J.-L. Pichard, "Gatemodulated thermopower of disordered nanowires: II. Variablerange hopping regime," New Journal of Physics, vol. 16, Article ID 095005, 2014.

[29] H. Abdalla, K. Van De Ruit, and M. Kemerink, "Effective temperature and universal conductivity scaling in organic semiconductors," Scientific Reports, vol. 5, Article ID 16870, 2015.

[30] L. F. Malmonge, S. Do Carmo Langiano, J. M. M. Cordeiro, L. H. C. Mattoso, and J. A. Malmonge, "Thermal and mechanical properties of PVDF/PANI blends," Materials Research, vol. 13, no. 4, pp. 465-470, 2010.

[31] K. Adrjanowicz, K. Kaminski, M. Dulski et al., "Dynamic glass transition and electrical conductivity behavior dominated by proton hopping mechanism studied in the family of hyperbranched Bis-MPA polyesters," Macromolecules , vol. 47, no. 16, pp. 5798-5807, 2014.

[32] R. Khazaka, M. L. Locatelli, S. Diaham, P. Bidan, L. Dupuy, and G. Grosset, "Broadband dielectric spectroscopy of BPDA/ODA 
polyimide films," Journal of Physics D: Applied Physics, vol. 46, no. 6, Article ID 065501, 2013.

[33] B. M. Greenhoe, M. K. Hassan, J. S. Wiggins, and K. A. Mauritz, "Universal power law behavior of the AC conductivity versus frequency of agglomerate morphologies in conductive carbon nanotube-reinforced epoxy networks," Journal of Polymer Science Part B: Polymer Physics, vol. 54, no. 19, pp. 1918-1923, 2016.

[34] B. Belaabed, S. Lamouri, N. Naar, P. Bourson, and S. O. S. Hamady, "Polyaniline-doped benzene sulfonic acid/epoxy resin composites: Structural, morphological, thermal and dielectric behaviors," Polymer Journal, vol. 42, no. 7, pp. 546-554, 2010.

[35] A. Choudhury, "Polyaniline/silver nanocomposites: dielectric properties and ethanol vapour sensitivity," Sensors and Actuators B: Chemical, vol. 138, no. 1, pp. 318-325, 2009.

[36] A. K. Jonscher, "Dielectric relaxation in solids," Journal of Physics D: Applied Physics, vol. 32, no. 14, pp. R57-R70, 1999.

[37] P. Sobolčiak, A. Ali, M. K. Hassan et al., "2D Ti3C2Tx (MXene)reinforced polyvinyl alcohol (PVA) nanofibers with enhanced mechanical and electrical properties," PLoS ONE, vol. 12, no. 8, Article ID e0183705, 2017. 


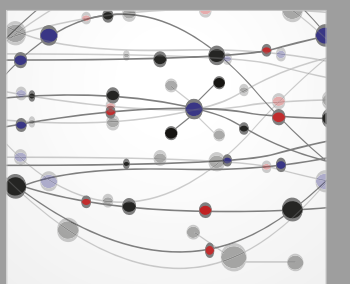

The Scientific World Journal
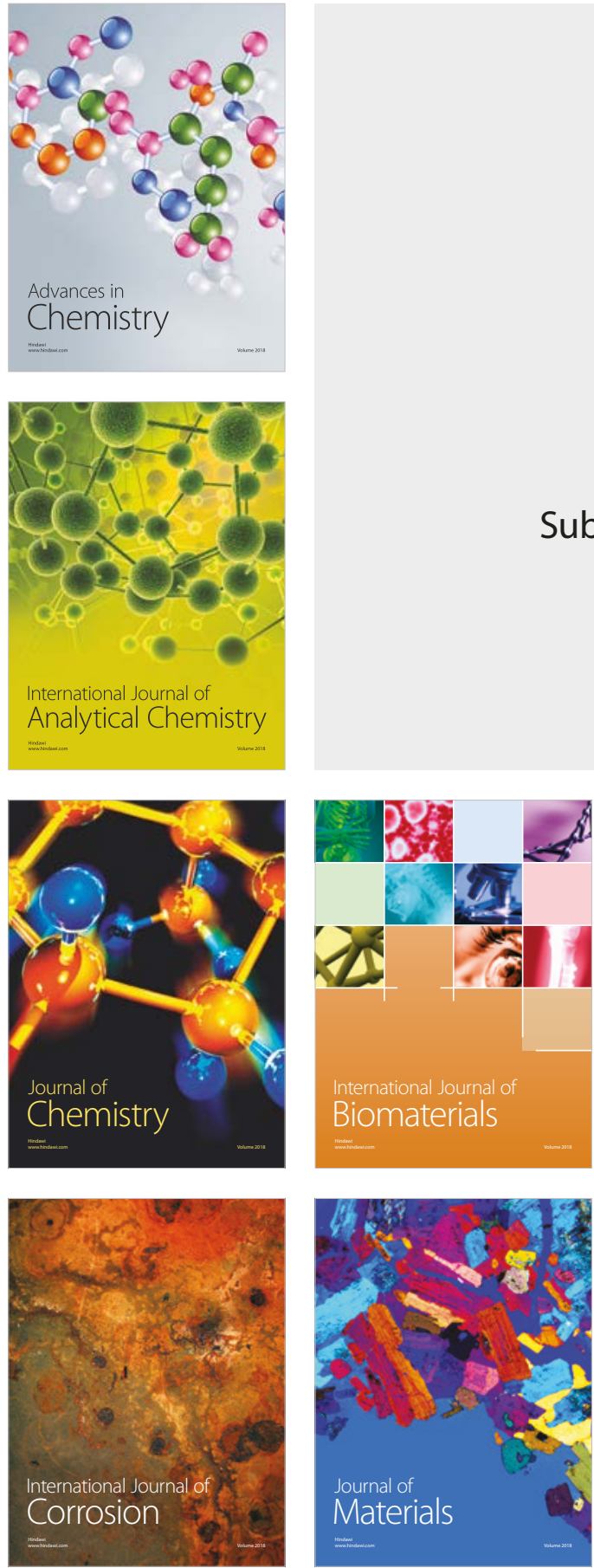

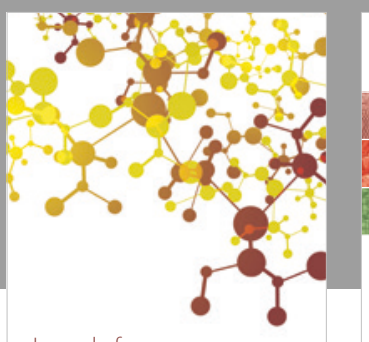

Journal of

Applied Chemistry
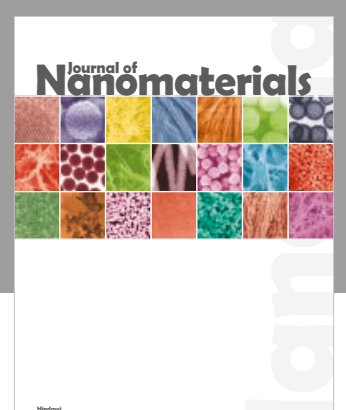

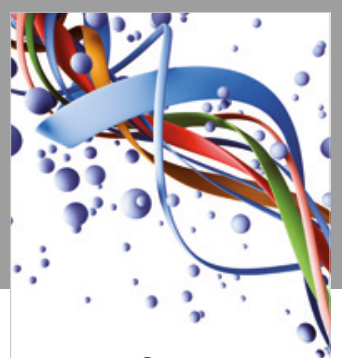

Scientifica

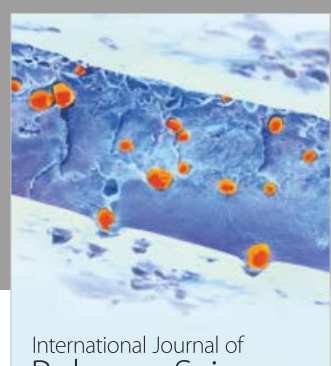

Polymer Science

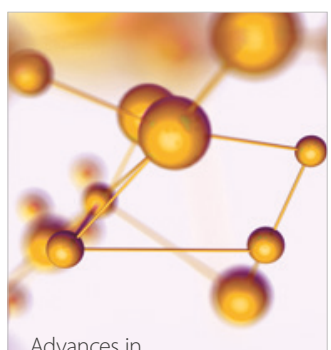

Physical Chemistry
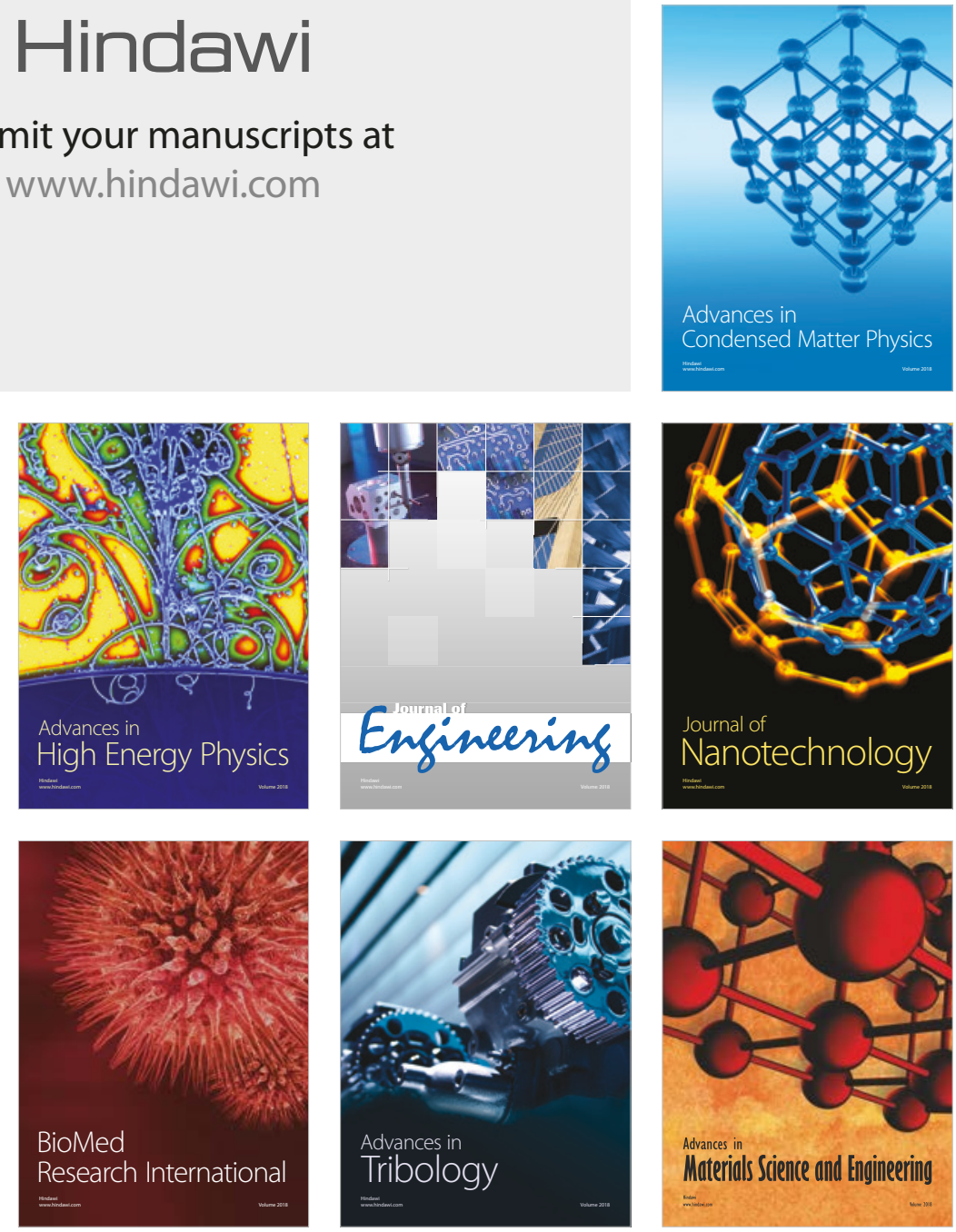